\title{
New Concepts for 3D Optics in X-ray Microscopy
}

\author{
Umut Tunca Sanli ${ }^{1}$, Hakan Ceylan ${ }^{2}$, Chengge Jiao ${ }^{3}$, Margarita Baluktsian ${ }^{1}$, Corinne Grevent ${ }^{1}$, \\ Kersten Hahn ${ }^{4}$, Yi Wang ${ }^{4}$, Vesna Srot ${ }^{4}$, Gunther Richter ${ }^{1}$, Iuliia Bykova ${ }^{1}$, Markus Weigand ${ }^{1}$, \\ Metin Sitti ${ }^{2}$, Gisela Schütz ${ }^{1}$, Kahraman Keskinbora ${ }^{1, *}$ \\ 1. Modern Magnetic Systems Department, Max Planck Institute for Intelligent Systems, \\ Stuttgart 70569, Germany \\ 2. Physical Intelligence Department, Max Planck Institute for Intelligent Systems, Stuttgart \\ 70569, Germany \\ 3. Thermo Fisher Scientific, 5651 GG Eindhoven, The Netherlands \\ 4. Stuttgart Center for Electron Microscopy, Max Planck Institute for Solid State Research, \\ Stuttgart 70569, Germany \\ * Corresponding author: keskinbora@is.mpg.de
}

Resolutions in the nanometer and sub-nanometer scale in XRM would enable an entirely new world of exciting science and strongly benefit life and earth sciences. Currently, there are two different strategies for improving the resolution of XRM. The first concept is related to the improvement of full-field or scanning transmission X-ray microscopy (STXM) and requires the realization of excellent high-resolution optics. The second approach is to promote ptychography, a combination of STXM and coherent diffractive imaging (CDI). Ptychography -in principle- allows reaching a wavelength-limited resolution but the resolution strongly depends on the incident coherent dose. Therefore, developing an optic with a high focusing efficiency remains essential for targeting resolutions in the nanometer and sub-nanometer scale [1].

We focus our research on developing different X-ray optics [2-5] to advance both conventional XRM and ptychography to their boundaries. In this light, our research does not target gradual improvements but focuses on innovating unorthodox strategies targeting the extreme and to pave the way for significant advancement.

The recent advancements in lithography instrumentation and sophisticated engineering solutions enabled the realization of binary Fresnel zone plates (FZP) of even smaller features. However, the achieved imaging resolutions via conventional XRM have converged to about 10 $\mathrm{nm}$ in half-pitch, and there has not been a significant improvement in the image resolution for almost a decade. Calculations based on the coupled wave theory (CWT) reveal that highly efficient focusing of X-rays to sub-10 $\mathrm{nm}$ can be achieved if the zones of the FZPs are tilted to the Bragg angle [6]. To realize tilted FZPs of high quality, we prepare micro-pillars with precise tilt angles using the recently developed plasma focused ion beam system (P-FIB). We then exploit the conformality of atomic layer deposition to deposit the multi-layer (ML) zones of the FZP over the tilted micro-pillars. Individual and precisely tilted ML-FZPs are then sliced, mounted on a sample holder grid, and polished using a $\mathrm{Ga}^{+}$FIB. We demonstrate that we can precisely tailor the tilt angle by controlling the PFIB milling parameters. Successful fabrication of the ML-FZPs, a thorough characterization of the zone quality and finally, yet importantly, direct imaging results reaching a $15 \mathrm{~nm}$ cut-off resolution at a soft X-ray STXM (MAXYMUS, BESSY II) has been achieved [6].

For advancing the resolution of the X-ray ptychography, we introduced new and advanced 3D Kinoform type optics $[4,7,8]$. Their focusing performance with respect to both resolution and efficiency were tested by direct ptychographic imaging using soft X-rays up to $2 \mathrm{keV}$ as the first step. In addition to metallic polycrystalline and amorphous media, we use low-loss soft 
materials to achieve extremely high focusing efficiencies. Our advanced, fast and cost-effective fabrication routes allow a faithful reproduction of the parabolic Kinoform profile while offering virtually unlimited optical thickness, hence can effectively target also high energy X-rays. A high measured focusing efficiency reaching $20 \%$ in soft X-rays match the theoretical expectations (reaching up to $95 \%$ of the theoretical values) proving the applicability of the suggested technique [8].

We are convinced that the proposed new strategies for making X-ray lenses will have a significant impact on direct imaging and ptychography experiments for both the synchrotronbased and laboratory-based X-ray tools.

\section{References:}

[1] Schropp, A. \& Schroer, C. G. Dose requirements for resolving a given feature in an object by coherent x-ray diffraction imaging. New J. Phys. 12 (2010), 035016.

[2] Keskinbora, K., et al. Rapid Prototyping of Fresnel Zone Plates via Direct Ga+ Ion Beam Lithography for High-Resolution X-ray Imaging. ACS Nano 7, 9788-9797 (2013).

[3] Sanli, U. T. et al. "High-resolution high-efficiency multilayer Fresnel zone plates for soft and hard x-rays." X-Ray Nanoimaging: Instruments and Methods II. Vol. 9592. International Society for Optics and Photonics, 2015.

[4] Sanli, U. T. et al. "Overview of the multilayer-Fresnel zone plate and the kinoform lens development at MPI for Intelligent Systems." EUV and X-ray Optics: Synergy between Laboratory and Space IV. Vol. 9510. International Society for Optics and Photonics, 2015.

[5] Keskinbora, K. et al. Multilayer Fresnel zone plates for high energy radiation resolve $21 \mathrm{~nm}$ features at 1.2 keV. Opt. Express 22, 18440-18453 (2014).

[6] Sanli, U. T. et al., 3D Nanofabrication of High-resolution Multilayer Fresnel Zone Plates. Adv. Sci. DOI:10.1002/advs.201800346 (2018).

[7] Keskinbora, K. et al. Single-Step 3D Nanofabrication of Kinoform Optics via Gray-Scale Focused Ion Beam Lithography for Efficient X-Ray Focusing. Adv. Opt. Mater. 3 (2015), p. $792-800$.

[8] Sanli, U. T. et al. 3D Nano-printed Plastic Kinoform X-ray Optics. Under Revision. (2018) 